\title{
An Enhanced Differential Surface Admittance Operator for the Signal Integrity Modeling of Interconnects
}

\author{
Martijn Huynen, Kamil Yavuz Kapusuz, Daniël De Zutter, Dries Vande Ginste \\ Electromagnetics Group/IDLab, Department of Information Technology \\ Ghent University/imec, Ghent, Belgium \\ martijn.huynen@ugent.be,kamilyavuz.kapusuz@UGent.be, daniel.dezutter@ugent.be,dries.vandeginste@ugent.be
}

\begin{abstract}
A new, enhanced formulation of the 3-D differential surface admittance operator is presented in this contribution. By employing closed expressions for the sums of the infinite series that arise from discretizing the operator by means of entire domain basis functions, a more efficient and accurate form is obtained. Convergence analysis demonstrates the performance gain. Additionally, the appositeness of the novel operator is studied by analyzing results for various interconnect structures over a broad frequency range and by comparing with other research and commercial solvers.
\end{abstract}

Index Terms-3-D surface admittance, boundary integral equation (BIE), interconnect modeling.

\section{INTRODUCTION}

Our modern society is characterized by an abundance of electronic devices and appliances that enables (wireless) connectivity on an unparalleled scale. Ever-increasing bandwidth requirements and integration of smart functionality in all aspects of our lives, have led to very diverse electronic systems in an expanding number of (new) technologies and applications. Of course, these trends and evolutions come with their specific challenges, which may include manufacturability, process inaccuracies and/or signal and power integrity (SI/PI). The latter two categories include well-known phenomena with potentially detrimental consequences such as crosstalk, signal attenuation and dispersion. To avoid costly redesigns at the end of the production process, it is therefore crucial for designers to assess the influence of these effects on the operation of the system as early as possible. To accommodate this need, fullwave computer-aided design tools have to continue evolving to stay accurate and relevant.

With the continuing miniaturization and increasing data rates, the need for full-blown three-dimensional (3-D) solvers that provide accurate, broadband results only rises. For interconnects, the correct modeling of the conductors is very challenging due to the field crowding associated with the skin effect. A correct broadband simulation of this skin effect is, however, of critical importance for the aforementioned SI analysis. While in volumetric methods this leads to an insurmountable number of unknowns, boundary-based methods struggle with the accurate numerical integration of the Green's function in the conductive medium [1]. Many techniques to solve or circumvent these obstacles have been developed over the years; a popular example being the surface impedance boundary conditions [2], which replaces the material inside by the background medium and enforces a local approximate relation to account for the material's influence. The differential surface admittance operator, first proposed in 2-D [3], on the other hand, provides a global relation that overcomes the shortcomings of the surface impedance conditions and enables accurate broadband modeling. Recently, a 3-D differential surface admittance operator has been proposed [4] that solves the interior problem based on the eigenmodes of the volume. In [4], [5], the broadband validity and accuracy of this method has been amply demonstrated but one major downside associated with the skin effect remains: a large number of eigenmodes are required for obtaining accurate results.

In this contribution, we propose a new formulation of the differential surface admittance operator for cuboids based on entire domain basis functions that, through closed sums of series, considerately improves the efficiency of its calculation, especially for the strongly developed skin effect. By comparison with commercial software tools and other approaches to interconnect modeling, we demonstrate the augmented efficiency and precision of this novel formulation.

\section{ENTIRE DOMAIN BASIS FUNCTIONS}

The 3-D differential surface admittance operator, as introduced for cuboids in [4], enables the replacement of a homogeneous, nonmagnetic material with wavenumber $k$ in a volume $\mathcal{V}$ by the nonmagnetic background material, characterized by its wavenumber $k_{0}$ through the introduction of an equivalent surface current density $\mathbf{j}_{s}$ on the boundary surface $\mathcal{S}$. This surface current density is related to the tangential electric field on the boundary, viz., $\mathbf{e}^{t}$, through the differential surface admittance operator $\mathcal{Y}$ as $\mathbf{j}_{s}=\mathcal{Y} \mathbf{e}^{t}$. An elegant way to construct this operator is based on the magnetic solenoidal eigenfunctions of $\mathcal{V}$, i.e., $\mathbf{h}_{\nu}$, [6]:

$$
\mathbf{j}_{s}=\mathcal{Y} \mathbf{e}^{t}=-\eta \sum_{\nu} \frac{\mathcal{K}_{\nu}}{\mathcal{N}_{\nu}^{2}}\left[\int_{\mathcal{S}}\left(\hat{\mathbf{n}} \times \mathbf{h}_{\nu}\right) \cdot \mathbf{e}_{t} \mathrm{~d} S\right]\left(\hat{\mathbf{n}} \times \mathbf{h}_{\nu}\right),
$$

with the contrast parameter $\eta=\left(k^{2}-k_{0}^{2}\right) / j \omega \mu_{0}, k_{\nu}$ and $\mathcal{N}_{\nu}$ the wavenumber and normalization constant of the eigenfunction $\mathbf{h}_{\nu}$, respectively, $\hat{\mathbf{n}}$ the outward pointing normal 
vector and $\mathcal{K}_{\nu}=k_{\nu}^{2} /\left(k_{0}^{2}-k_{\nu}^{2}\right) /\left(k^{2}-k_{\nu}^{2}\right)$. To facilitate integration of this operator $\mathcal{Y}$ into a method to solve the exterior problem, e.g., the Method of Moments (MoM), it needs to be discretized. Expanding both $\mathbf{j}_{s}$ and $\mathbf{e}^{t}$ into basis functions $\mathbf{f}_{i}(\mathbf{r})$, transforms (1) into

$$
\overline{\bar{G}} \mathbf{I}=\overline{\bar{Y}} \mathbf{E},
$$

where the vectors $\mathbf{I}$ and $\mathbf{E}$ contain the expansions coefficients of $\mathbf{j}_{s}$ and $\mathbf{e}^{t}$, respectively, while the elements of the Gram matrix $\bar{G}$ and the discretized differential surface admittance operator $\bar{Y}$ are given by

$$
\begin{aligned}
& (\bar{G})_{i j}=\int_{\mathcal{S}_{i}} \mathbf{f}_{i}(\mathbf{r}) \cdot \mathbf{f}_{j}(\mathbf{r}) \mathrm{d} S \\
& (\bar{Y})_{i j}=-\eta \sum_{\nu} \frac{\mathcal{K}_{\nu}}{\mathcal{N}_{\nu}^{2}} \int_{\mathcal{S}_{i}}\left(\hat{\mathbf{n}} \times \mathbf{h}_{\nu}\right) \cdot \mathbf{f}_{i} \mathrm{~d} S \int_{\mathcal{S}_{j}}\left(\hat{\mathbf{n}} \times \mathbf{h}_{\nu}\right) \cdot \mathbf{f}_{j} \mathrm{~d} S,
\end{aligned}
$$

with $\mathcal{S}_{i}$ the support of basis function $\mathbf{f}_{i}$. Previously, we employed local functions such as RWGs or rooftops for this discretization [4]-[6], depending on the nature of $\mathcal{V}$. However, it turns out that the employment of entire domain basis functions on the faces of a cuboid enables a considerable improvement in terms of accuracy and computation time. For a proper choice of these basis functions, we first turn to the eigenfunctions $\mathbf{h}_{\nu}$ of the cuboid. These consists of two sets: transverse magnetic (TM) and transverse electric (TE) modes, which share the same wavenumber:

$k_{\nu}^{2}=k_{m n p}^{2}=\lambda_{x}^{2}+\lambda_{y}^{2}+\lambda_{z}^{2}=\left(\frac{m \pi}{l_{x}}\right)^{2}+\left(\frac{n \pi}{l_{y}}\right)^{2}+\left(\frac{p \pi}{l_{z}}\right)^{2}$,

with $\left(l_{x}, l_{y}, l_{z}\right)$ the dimensions of the cuboid and $\nu=(m, n, p)$ the triple index defined over the nonnegative integers.

The magnetic TM eigenfunctions themselves are expressed as

$$
\begin{aligned}
\mathbf{h}_{\nu}^{\mathrm{TM}} & =k_{\nu} \lambda_{y} \sin \left(\lambda_{x} x\right) \cos \left(\lambda_{y} y\right) \cos \left(\lambda_{z} z\right) \hat{\mathbf{x}} \\
& -k_{\nu} \lambda_{x} \cos \left(\lambda_{x} x\right) \sin \left(\lambda_{y} y\right) \cos \left(\lambda_{z} z\right) \hat{\mathbf{y}}
\end{aligned}
$$

while the TE modes are given by

$$
\begin{aligned}
\mathbf{h}_{\nu}^{\mathrm{TE}} & =\lambda_{z} \lambda_{x} \sin \left(\lambda_{x} x\right) \cos \left(\lambda_{y} y\right) \cos \left(\lambda_{z} z\right) \hat{\mathbf{x}} \\
& +\lambda_{z} \lambda_{y} \cos \left(\lambda_{x} x\right) \sin \left(\lambda_{y} y\right) \cos \left(\lambda_{z} z\right) \hat{\mathbf{y}} \\
& -\left(\lambda_{x}^{2}+\lambda_{y}^{2}\right) \cos \left(\lambda_{x} x\right) \cos \left(\lambda_{y} y\right) \sin \left(\lambda_{z} z\right) \hat{\mathbf{z}} .
\end{aligned}
$$

Both sets of eigenfunctions are not normalized but their normalization constant can easily be calculated as

$$
\begin{aligned}
\mathrm{TM}: \mathcal{N}_{\nu}^{2} & =k_{\nu}^{2} \frac{V}{4}\left(\lambda_{x}^{2}+\lambda_{y}^{2}\right) \frac{1}{\varepsilon_{p}} \\
\mathrm{TE}: \mathcal{N}_{\nu}^{2} & =k_{\nu}^{2} \frac{V}{2}\left(\lambda_{x}^{2}+\lambda_{y}^{2}\right) \frac{1}{\varepsilon_{m} \varepsilon_{n}}
\end{aligned}
$$

with the volume of $\mathcal{V}$ denoted as $V=l_{x} l_{y} l_{z}$ and $\varepsilon_{i}$ the Neumann factor [7], which equals 1 for $i=0$ and 2 otherwise.

In (4), the (rotated) tangential component of the eigenfunctions on each face of the cuboid pops up. Numbering these faces from zero to five, corresponding to their location at $x=0, x=l_{x}, y=0, y=l_{y}, z=0, z=l_{z}$, respectively, we take a closer look at the situation on face $S_{5}(\hat{\mathbf{n}}=\hat{\mathbf{z}})$ :

$$
\begin{aligned}
S_{5}: \hat{\mathbf{z}} \times \mathbf{h}_{\nu} & =\xi_{x, \nu}(-1)^{p} \sin \left(\lambda_{x} x\right) \cos \left(\lambda_{y} y\right) \hat{\mathbf{y}} \\
& -\xi_{y, \nu}(-1)^{p} \sin \left(\lambda_{y} y\right) \cos \left(\lambda_{x} x\right) \hat{\mathbf{x}},
\end{aligned}
$$

where $\xi_{\alpha, \nu}$ is a substitute for the factor of the $\alpha$-component of either the TM or TE modes, where $\alpha$ stands for one of the Cartesian axes. Note that both components of this term have a similar structure: notwithstanding the constant factor upfront, it is formed by the product of two trigonometric functions, i.e., a cosine function along its axis and a sine dependence on the other coordinate axis of the face. Inspection of the same quantity on the other five faces reveals the same regularity.

Taking this finding into account, we have found the candidates for the entire domain basis functions. Focusing first on these functions along the $x$-axis on $S_{5}$, they are defined as

$$
\mathbf{f}_{m^{\prime} n^{\prime}}^{x}=\cos \left(\lambda_{x}^{\prime} x\right) \sin \left(\lambda_{y}^{\prime} y\right) \hat{\mathbf{x}},
$$

with $m^{\prime}$ and $n^{\prime}$ the equivalent of indices $m$ and $n$ for the eigenmodes and with $\lambda_{x}^{\prime}$ and $\lambda_{y}^{\prime}$ fulfilling the same function as their nonprimed counterparts. The relevant integral in the calculation of the Gram matrix is now found to be

$$
\int_{S_{5}} \mathbf{f}_{m^{\prime} n^{\prime}}^{x} \cdot \mathbf{f}_{m^{\prime \prime} n^{\prime \prime}}^{x} \mathrm{~d} S=\frac{A_{5} \sigma_{n^{\prime}}}{2 \varepsilon_{m^{\prime}}} \delta_{m^{\prime} m^{\prime \prime}} \delta_{n^{\prime} n^{\prime \prime}},
$$

with $A_{5}$ the area of face $S_{5}, \delta_{i j}$ the Kronecker delta, $\sigma_{i}$ equal to zero for $i=0$ and one otherwise. Owing to the Kronecker deltas, the only nonzero entries for the $x$-directed basis functions on $S_{5}$ will be those for which $m^{\prime}=m^{\prime \prime}$ and $n^{\prime}=n^{\prime \prime}$. As this is true for the $y$-direction as well and furthermore generalizes to other faces, the Gram matrix for the entire domain functions will be a diagonal matrix.

Turning our attention to the $\bar{Y}$-matrix, the integrals involved are the same as (12) since the entire domain basis functions are of the same form as the rotated magnetic eigenfunctions. For two $x$-directed functions on $S_{5}$, we thus get

$$
-\eta \sum_{m n p} \frac{\mathcal{K}_{\nu}}{\mathcal{N}_{\nu}^{2}} \xi_{y, \nu}^{2}\left(\frac{A_{5} \sigma_{n}}{2 \varepsilon_{m}}\right)^{2} \delta_{m m^{\prime}} \delta_{n n^{\prime}} \delta_{m m^{\prime \prime}} \delta_{n n^{\prime \prime}}
$$

Similarly to the elements of the Gram matrix, the Kronecker deltas force the indices $(m, n)$ and $\left(m^{\prime \prime}, n^{\prime \prime}\right)$, respectively, to the same value as the first $x$-directed basis function, i.e., $\left(m^{\prime}, n^{\prime}\right)$. As such, the function $\mathbf{f}_{m^{\prime} n^{\prime}}^{x}$ maps to only one other $x$-directed basis function on $S_{5}$, i.e., itself, as schematically shown in Fig. 1. The coefficient is given by

$$
A_{m^{\prime} n^{\prime}}^{x x}=\frac{A_{5} \sigma_{n^{\prime}}}{2 \varepsilon_{m^{\prime}}}\left[-\eta \sum_{p=0}^{\infty} \frac{\mathcal{K}_{m^{\prime} n^{\prime} p}}{\mathcal{N}_{m^{\prime} n^{\prime} p}^{2}} \xi_{y, m^{\prime} n^{\prime} p}^{2}\right] \frac{A_{5} \sigma_{n^{\prime}}}{2 \varepsilon_{m^{\prime}}}
$$

The same basis function maps onto a second one on $S_{5}$, viz., $\mathbf{f}_{m^{\prime} n^{\prime}}^{y}$ the $y$-directed equivalent of (11), as shown in Fig. 1. 


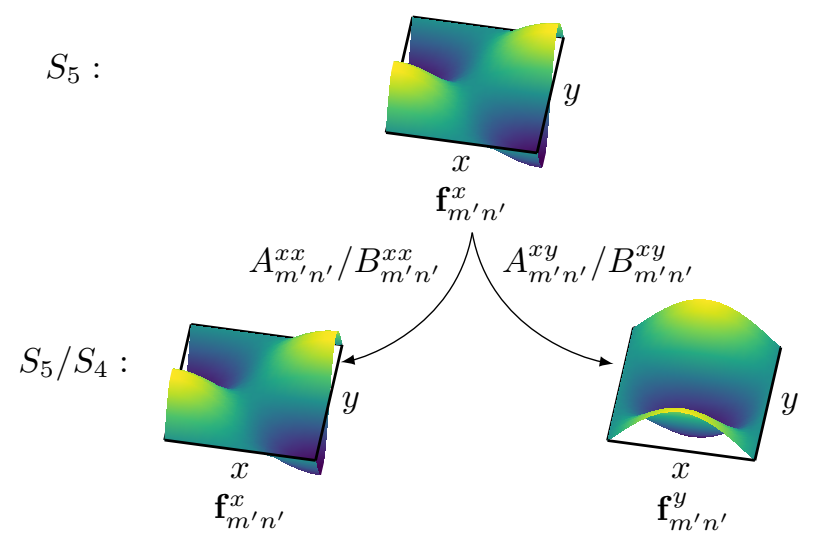

Fig. 1. Schematic representation of the structure of the $\overline{\bar{Y}}$-matrix elements for one basis functions on $S_{5}$ and the other on either $S_{5}$ or $S_{4}$. In this illustration $m^{\prime}$ and $n^{\prime}$ are set to 1 and 2, respectively.

Following similar steps as outlined above, the relevant $\bar{Y}$ element is given by

$$
A_{m^{\prime} n^{\prime}}^{x y}=\frac{A_{5} \sigma_{m^{\prime}}}{2 \varepsilon_{n^{\prime}}}\left[\eta \sum_{p=0}^{\infty} \frac{\mathcal{K}_{m^{\prime} n^{\prime} p}}{\mathcal{N}_{m^{\prime} n^{\prime} p}^{2}} \xi_{x, m^{\prime} n^{\prime} p} \xi_{y, m^{\prime} n^{\prime} p}\right] \frac{A_{5} \sigma_{n^{\prime}}}{2 \varepsilon_{m^{\prime}}} .
$$

The reach of $\mathbf{f}_{m^{\prime} n^{\prime}}^{x}$ on $S_{5}$ is not limited to its own face but extends to the other faces as well. First looking at the opposite face $S_{4}$, the same two basis functions $\mathbf{f}_{m^{\prime} n^{\prime}}^{x}$ and $\mathbf{f}_{m^{\prime} n^{\prime}}^{y}$ are excited with coefficients (as shown in Fig. 1):

$$
\begin{aligned}
B_{m^{\prime} n^{\prime}}^{x x}=\frac{A_{4} \sigma_{n^{\prime}}}{2 \varepsilon_{m^{\prime}}}\left[\eta \sum_{p=0}^{\infty}(-1)^{p} \frac{\mathcal{K}_{m^{\prime} n^{\prime} p}}{\mathcal{N}_{m^{\prime} n^{\prime} p}^{2}} \xi_{y, m^{\prime} n^{\prime} p}^{2}\right] \frac{A_{5} \sigma_{n^{\prime}}}{2 \varepsilon_{m^{\prime}}}, \\
B_{m^{\prime} n^{\prime}}^{x y}=\frac{A_{4} \sigma_{m^{\prime}}}{2 \varepsilon_{n^{\prime}}}\left[-\eta \sum_{p=0}^{\infty}(-1)^{p} \frac{\mathcal{K}_{m^{\prime} n^{\prime} p}}{\mathcal{N}_{m^{\prime} n^{\prime} p}^{2}} \times\right. \\
\left.\xi_{x, m^{\prime} n^{\prime} p} \xi_{y, m^{\prime} n^{\prime} p}\right] \frac{A_{5} \sigma_{n^{\prime}}}{2 \varepsilon_{m^{\prime}}} .
\end{aligned}
$$

Turning our attention to one of the remaining faces, e.g., $S_{3}$, the entire domain basis functions on this face are directed along either the $x$ - or $z$-axis. Focusing on the $z$-directed $\mathbf{f}_{m^{\prime \prime}}^{z} p^{\prime \prime}$ functions, the equivalent of (13) becomes

$$
-\eta \sum_{m n p} \frac{\mathcal{K}_{\nu}}{\mathcal{N}_{\nu}^{2}} \xi_{y, \nu} \xi_{x, \nu} \frac{A_{5} \sigma_{n}}{2 \varepsilon_{m}} \frac{A_{3} \sigma_{m}}{2 \varepsilon_{p}} \delta_{m m^{\prime}} \delta_{n n^{\prime}} \delta_{m m^{\prime \prime}} \delta_{p p^{\prime \prime}}
$$

Once again, the expression can be simplified into

$C_{m^{\prime} n^{\prime} p^{\prime \prime}}^{x z}=\frac{A_{3} \sigma_{m^{\prime}}}{2 \varepsilon_{p^{\prime \prime}}}\left[-\eta \frac{\mathcal{K}_{m^{\prime} n^{\prime} p^{\prime \prime}}}{\mathcal{N}_{m^{\prime} n^{\prime} p^{\prime \prime}}^{2}} \xi_{y, m^{\prime} n^{\prime} p^{\prime \prime}} \xi_{x, m^{\prime} n^{\prime} p^{\prime \prime}}\right] \frac{A_{5} \sigma_{n^{\prime}}}{2 \varepsilon_{m^{\prime}}}$

Remark that in contrast to (14)-(17), the coefficient still depends on one of the indices of the second basis functions, i.e., in this case $p^{\prime \prime}$. This implies that, contrary to the basis functions on parallel or coinciding faces, there is no longer a one-to-one relation between the basis functions on perpendicular faces. As demonstrated in Fig. 2, one single basis

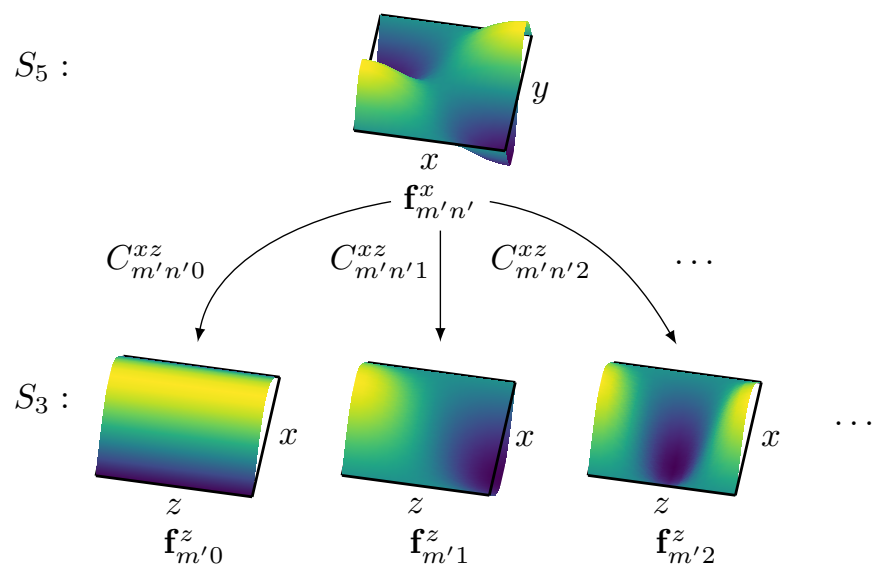

Fig. 2. Schematic representation of the structure of the $\overline{\bar{Y}}$-matrix elements for one basis functions on $S_{5}$ and the others on $S_{3}$. In this illustration $\mathrm{m}^{\prime}$ and $n^{\prime}$ are set to 1 and 2 , respectively.

function along the $x$-axis on $S_{5}$ excites an infinite series of entire domain basis functions on $S_{3}$ along the $z$-axis. The same procedure applied to the $x$-directed basis functions on $S_{3}$ and to all other entire domain basis function on the remaining faces leads to similar coefficients as (19).

At this point, we have to repeat the entire procedure detailed for $\mathbf{f}_{m^{\prime} n^{\prime}}^{x}$ on $S_{5}$ to all other entire domain basis functions and faces of the cuboid. Evidently, this will lead to completely analogous results as the ones described above given the proper permutation of the employed indices and axes. In the end, we collect all elements into various matrices, transforming (2) into

$$
\bar{G} \mathbf{I}=\bar{G} \bar{X} \bar{G} \mathbf{E} \Leftrightarrow \mathbf{I}=\bar{X} \bar{G} \mathbf{E},
$$

where the elements of $\bar{X}$ correspond to the bracketed terms in (14)-(19). The decomposed form of $\bar{Y}$ presented in (20) does not provide any inherent advantage over a discretization with local basis functions such as rooftops since (14)-(17) still contain series that have to be approximated numerically. However, we show in the next section that these series do in fact have closed forms for their sums.

\section{ClOSED-FORM EXPRESSIONS FOR THE SUM OF SERIES}

We first take a look at the series present in (14). Keeping in mind that the contributions for both the TM and TE modes should be taken into account, the bracketed term in (14) becomes

$$
\begin{aligned}
& -\frac{4 \eta}{V}\left(\sum_{p=0}^{\infty} \frac{\epsilon_{p} k_{m^{\prime} n^{\prime} p}^{2} \lambda_{x}^{\prime 2}}{\left(k^{2}-k_{m^{\prime} n^{\prime} p}^{2}\right)\left(k_{0}^{2}-k_{m^{\prime} n^{\prime} p}^{2}\right)\left({\lambda^{\prime}}_{x}^{2}+{\lambda^{\prime}}_{y}^{2}\right)}\right. \\
& \left.\quad+\epsilon_{m} \sum_{p=1}^{\infty} \frac{{\lambda_{y}^{\prime}}_{y}^{2}{\lambda^{\prime}}_{z}^{2}}{\left(k^{2}-k_{m^{\prime} n^{\prime} p}^{2}\right)\left(k_{0}^{2}-{k_{m}^{\prime} n^{\prime} p}^{2}\right)\left(\lambda_{x}^{\prime 2}+\lambda_{y}^{\prime 2}\right)}\right) .
\end{aligned}
$$


Recombining both sums, extracting the $p=0$ term from the first series and implementing the following substitutions:

$$
\begin{gathered}
\gamma=\frac{l_{z}}{\pi} \tilde{\gamma}=\frac{l_{z}}{\pi} \sqrt{k^{2}-{\lambda^{\prime}}_{x}^{2}-\lambda^{\prime 2}} \\
\gamma_{0}=\frac{l_{z}}{\pi} \tilde{\gamma}_{0}=\frac{l_{z}}{\pi} \sqrt{k_{0}^{2}-{\lambda^{\prime}}_{x}^{2}-{\lambda^{\prime}}_{y}^{2}} \\
\rho=\frac{l_{z}}{\pi} \lambda^{\prime}{ }_{x}=m^{\prime} l_{z} / l_{x} \quad \varrho=\frac{l_{z}}{\pi} \lambda_{y}^{\prime}=n^{\prime} l_{z} / l_{y}
\end{gathered}
$$

we rewrite (21) as

$$
-\frac{4 \eta}{V} \frac{l_{z}^{2}}{\pi^{2}}\left(\frac{\rho^{2}}{\gamma^{2} \gamma_{0}^{2}}+\frac{\varepsilon_{m^{\prime}}}{\gamma^{2}-\gamma_{0}^{2}}\left[\sum_{p=1}^{\infty} \frac{\rho^{2}+\gamma^{2}}{p^{2}-\gamma^{2}}-\frac{\rho^{2}+\gamma_{0}^{2}}{p^{2}-\gamma_{0}^{2}}\right]\right) .
$$

The two sums in (25) are both of the same form and have a closed sum expression [8]:

$$
\sum_{k=1} \frac{1}{k^{2}-\alpha^{2}}=\frac{1}{2 \alpha^{2}}-\frac{\pi}{2 \alpha} \cot (\pi \alpha),
$$

which leads to the following expression for (25):

$$
\frac{2 \varepsilon_{m^{\prime}} l_{z}}{j \omega \mu_{0} V}\left(\frac{k^{2}-\lambda_{y}^{\prime 2}}{\tilde{\gamma}} \cot (\pi \gamma)-\frac{k_{0}^{2}-\lambda_{y}^{\prime 2}}{\tilde{\gamma}_{0}} \cot \left(\pi \gamma_{0}\right)\right) \text {. }
$$

The series in (15) can be resolved in a similar manner, resulting in

$$
\frac{4 l_{z}}{j \omega \mu_{0} V} \lambda^{\prime}{ }_{x} \lambda^{\prime}{ }_{y}\left(\frac{1}{\tilde{\gamma}} \cot (\pi \gamma)-\frac{1}{\tilde{\gamma}_{0}} \cot \left(\pi \gamma_{0}\right)\right) .
$$

Note that the series in (16) and (17) are the same as the ones in (14) and (15) apart from a factor $-(-1)^{p}$. This transforms the infinite sums in the equivalent of (25) for (16) into the following expression with its own closed sum [9]:

$$
\sum_{k=1} \frac{(-1)^{k}}{k^{2}-\alpha^{2}}=\frac{1}{2 \alpha^{2}}-\frac{\pi}{2 \alpha} \csc (\pi \alpha) .
$$

In the end, we thus find the following evaluations for the bracketed terms in (16) and (17), respectively:

$$
\begin{gathered}
-\frac{2 \varepsilon_{m^{\prime}} l_{z}}{j \omega \mu_{0} V}\left(\frac{k^{2}-{\lambda^{\prime}}_{y}^{2}}{\tilde{\gamma}} \csc (\pi \gamma)-\frac{k_{0}^{2}-{\lambda^{\prime}}_{y}^{2}}{\tilde{\gamma}_{0}} \csc \left(\pi \gamma_{0}\right)\right) \\
-\frac{4 l_{z}}{j \omega \mu_{0} V} \lambda^{\prime}{ }_{x} \lambda^{\prime}{ }_{y}\left(\frac{1}{\tilde{\gamma}} \csc (\pi \gamma)-\frac{1}{\tilde{\gamma}_{0}} \csc \left(\pi \gamma_{0}\right)\right)
\end{gathered}
$$

An extensions of this approach to all other, relevant elements in $\bar{X}$ is achieved, due to symmetry, by the correct cyclic permutation of the indices and axes. In the end, this replaces any series present in $\bar{X}$ by closed analytical expressions and thus results in a more efficient and accurate formulation.

\section{EXAMPLES}

As a first example, we study the convergence behavior of the series in (14) and (16) for an increasing number of terms. To this end, we compare the values of $A_{m^{\prime} n^{\prime}}^{x x}$ and $B_{m^{\prime} n^{\prime}}^{x x}$ to the ones calculated by means of the closed sums (27) and (30) for $m^{\prime}=n^{\prime}=1$ (other values of $m^{\prime}$ and $n^{\prime}$ behave similarly). These are computed for a cube with a side of $1 \mathrm{~mm}$ for two different materials: a dielectric with $\epsilon_{r}=4$ and copper with $\sigma=5.8 \cdot 10^{7} \mathrm{~S} / \mathrm{m}$. The evolution of the relative errors is demonstrated in Fig. 3(a) for a relatively low frequency of $1 \mathrm{kHz}$. We observe that this error descends slower for $A_{11}^{x x}$ than for $B_{11}^{x x}$ for both materials. Moreover, the relative errors for the dielectric and the copper display the same convergence behavior. At this frequency, the skin depth of copper is about twice the size of the cube and as such behaves similar to a lossless dielectric. If we increase the frequency, the skin effect kicks in and this influences the relative error. At $100 \mathrm{kHz}$, where the skin depth is about a fifth of the cube's size, we see in Fig. 3(b) that the convergence for the copper values starts to deteriorates while the error values for the dielectric remain undisturbed. At $1 \mathrm{GHz}$ with a fully developed skin effect, the number of terms required to attain the same relative errors for the copper cube as for the lower frequencies has increased with two orders of magnitude, as shown in Fig. 3(c). The closed expressions for the sums of the series in (14) and (16) thus provide a substantial and clear accuracy improvement that furthermore increases the efficiency of the differential surface admittance operator.

Next, we examine the interconnect structure depicted in Fig. 4 consisting of two sets of three parallel conductors with a conductivity of $3.57 \cdot 10^{7} \mathrm{~S} / \mathrm{m}$ [10]. All six lines have a length of $100 \mu \mathrm{m}$ with a cross-section of $6 \mu \mathrm{m} \times 4 \mu \mathrm{m}$. The structure is simulated by integrating the enhanced differential surface admittance operator into an augmented EFIE formulation [5], [11], resulting in a broadband characterization of the impedance matrix, which is compared to the reference result presented in [10]. In Fig. 5, some relevant matrix elements are plotted, where the markers represent the reference solution while the various lines correspond to the differential surface admittance operator. Note that both the real and imaginary part of $Z_{25}$ are, in fact, nonzero but several orders of magnitude smaller than the other selected impedance matrix elements. Overall, we observe excellent agreement between both methods. Slight deviations are present (most notably for $Z_{11}$ and $Z_{13}$ in Fig. 5(b)) that can be attributed to the assumptions made in constructing the equivalent surface impedance in [10] and, potentially, the difference in port definition.

Consider now the RF coil shown in Fig. 6 [12]. The copper conductors $\left(\sigma=5.8 \cdot 10^{7} \mathrm{~S} / \mathrm{m}\right)$ constituting the coil have a square cross-section with a side of $3 \mu \mathrm{m}$. The coil has three windings separated by a $1 \mu \mathrm{m}$ spacing with the longest sides measuring $40 \mu \mathrm{m}$. Its port is defined across the $1 \mu \mathrm{m}$ air gap present in the middle of the underpass. More details about the geometry can be found annotated on Fig. 6. The structure is simulated employing the enhanced differential 


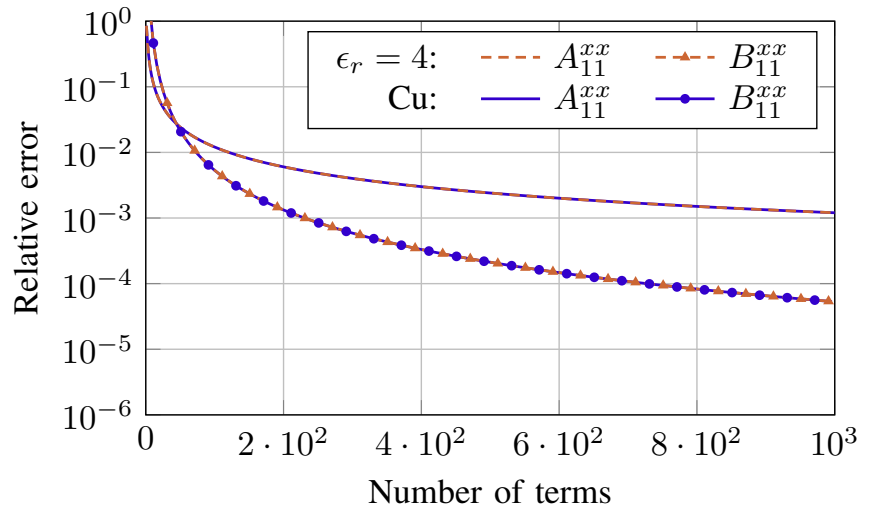

(a) $f=1 \mathrm{kHz}$.

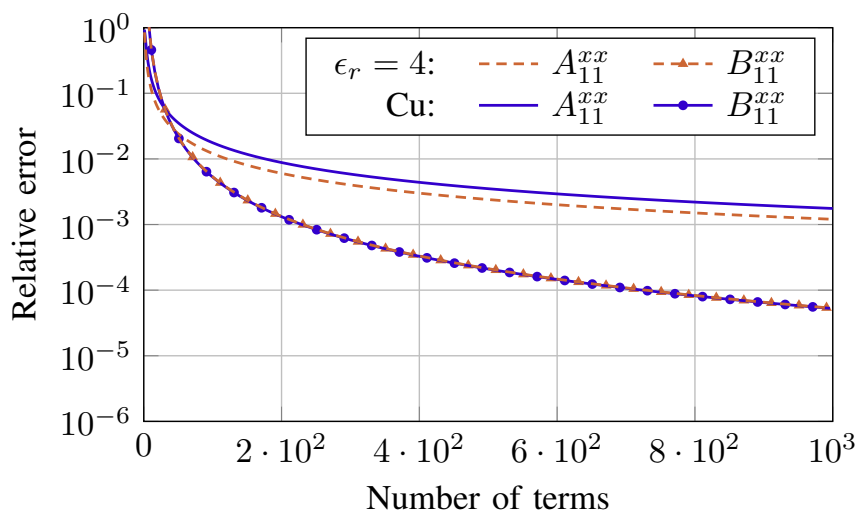

(b) $f=100 \mathrm{kHz}$.

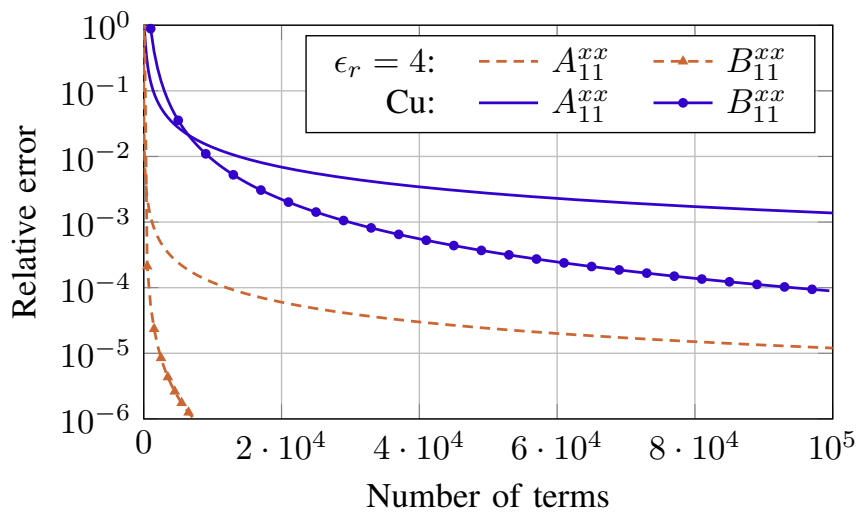

(c) $f=1 \mathrm{GHz}$.

Fig. 3. Relative error of $A_{11}^{x x}$ and $B_{11}^{x x}$ as a function of the number of terms in their series as compared to the sums (27) and (30), respectively, for a low-contrast dielectric $\left(\epsilon_{r}=4\right)$ and copper $\left(5.8 \cdot 10^{7} \mathrm{~S} / \mathrm{m}\right)$ at three different frequencies.

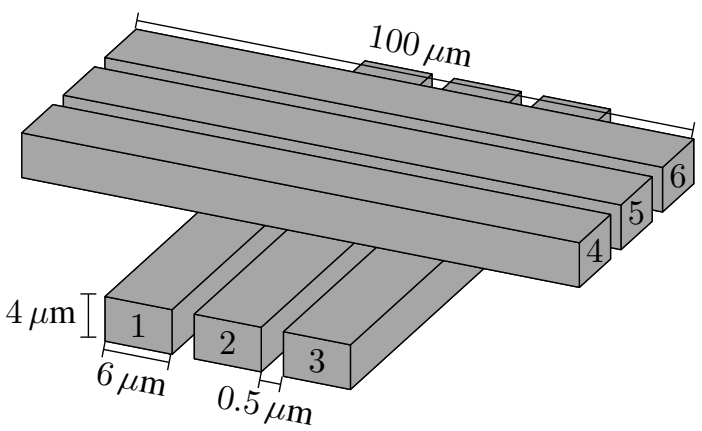

Fig. 4. Two sets of three parallel conductors $\left(\sigma=3.57 \cdot 10^{7} \mathrm{~S} / \mathrm{m}\right)$. The vertical spacing between the two sets is $0.5 \mu \mathrm{m}$, i.e., the same distance as present between the parallel conductors [10].

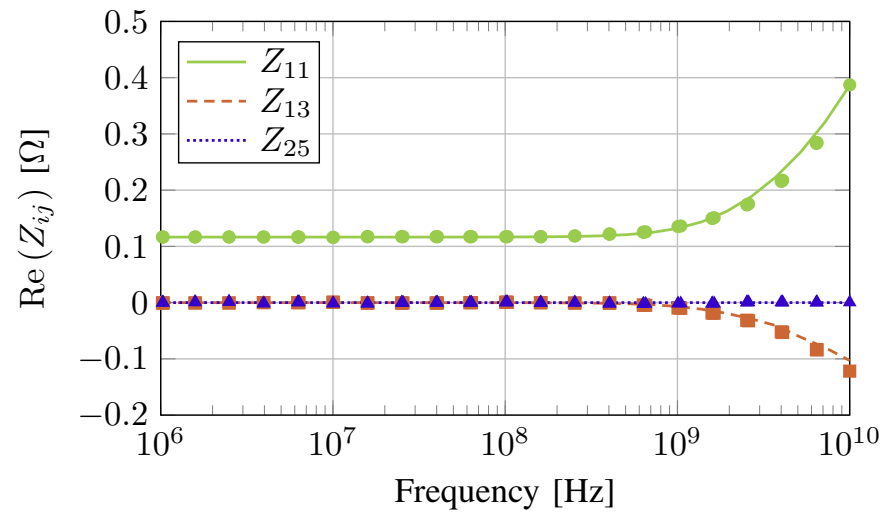

(a) Real part of $Z_{i j}$ in $\Omega$.

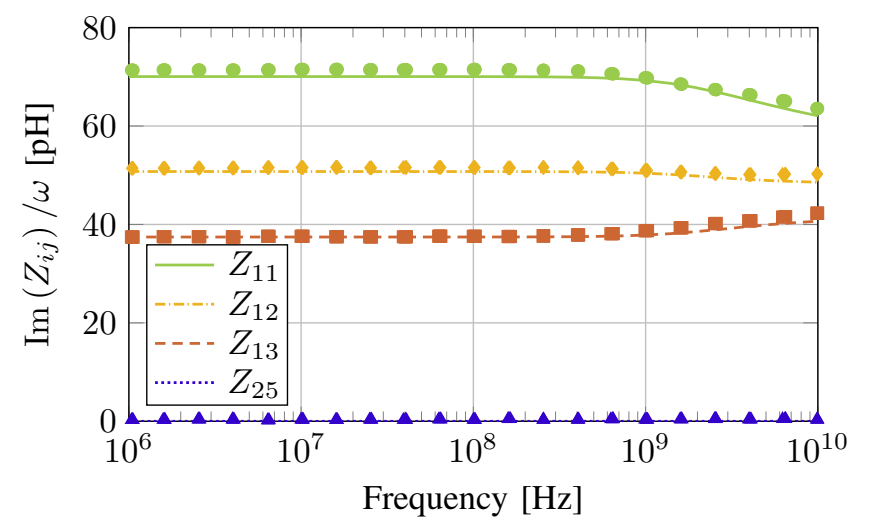

(b) Imaginary part of $Z_{i j}$ over $\omega$ in $\mathrm{pH}$.

Fig. 5. Selected impedance matrix elements of the interconnect structure depicted in Fig. 4. Results from this work are plotted with lines while the results from [10] are shown with markers. 


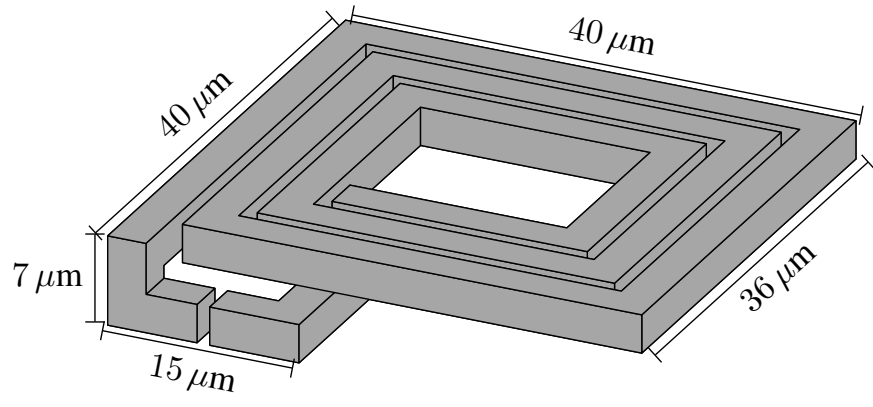

Fig. 6. Copper $\left(\sigma=5.8 \cdot 10^{7} \mathrm{~S} / \mathrm{m}\right) \mathrm{RF}$ coil with a square cross-section of $3 \mu \mathrm{m} \times 3 \mu \mathrm{m}$. The different windings are separated by a $1 \mu \mathrm{m}$ spacing. A port is defined over the $1 \mu \mathrm{m}$ air gap in the middle of the underpass [12].

surface admittance operator and its results are compared to the commercial solvers CST Microwave Studio (MWS) [13] and ANSYS HFSS [14]. The resulting impedance is shown in Fig. 7. Focusing first on the real part in Fig. 7(a), all three curves coincide for low-frequency values. Once the frequency increases and current crowding inside the conductors comes into play, a difference between the solvers is clearly observed. Both reference solvers, which employ volume based methods, exhibit a faster increasing resistance as the mesh refinement start to struggle with the rapidly varying fields inside the material; a drawback not present for the differential surface admittance operator as the fields inside are not computed directly, but are taken into account via the equivalent surface current density. The low-frequency values of the inductance, presented in Fig. 7(b), differ for all three solvers. For the commercial solvers, the value is sensitive to the bounding box dimensions and imposed boundary conditions to approximate free space while the BIE-based method presented here takes the unboundedness into account automatically through use of the free-space Green's function. For higher frequencies, the same remarks can be made as for the resistance, i.e., the volume meshing results in a faster decline of the inductance.

\section{CONCLUSION}

An enhanced formulation of the 3-D differential surface admittance operator for cuboids is introduced. Through the use of entire domain basis functions and closed forms for the sums of the resulting series, an important downside of previous expressions of this operator is alleviated, resulting in a substantial increase in accuracy and efficiency. This improvement is demonstrated by studying the convergence behavior of the matrix elements and the differential surface admittance operator is employed to characterize interconnect structures in comparison to commercial software and similar methods.

\section{REFERENCES}

[1] J. Peeters, I. Bogaert, and D. De Zutter, "Calculation of MoM interaction integrals in highly conductive media," IEEE Trans. Antennas Propag., vol. 60, no. 2, pp. 930-940, Feb. 2012.

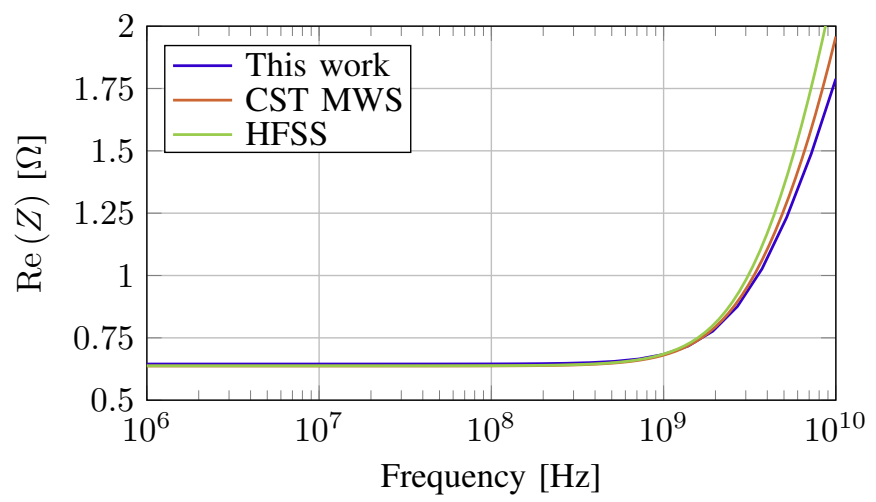

(a) Real part of $Z$ in $\Omega$.

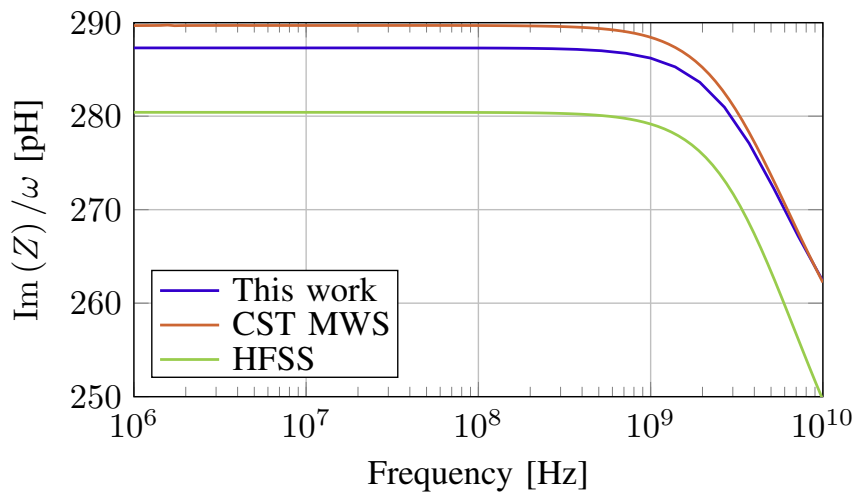

(b) Imaginary part of $Z$ over $\omega$ in $\mathrm{pH}$.

Fig. 7. Impedance $Z$ of the copper coil depicted in Fig. 6.

[2] T. Senior and J. Volakis, Approximate Boundary Conditions in Electromagnetics, ser. Electromagnetics and Radar Series. Institution of Electrical Engineers, 1995.

[3] D. De Zutter and L. Knockaert, "Skin effect modeling based on a differential surface admittance operator," IEEE Trans. Microw. Theory Techn., vol. 53, no. 8, pp. 2526-2538, Aug. 2005.

[4] M. Huynen, D. De Zutter, and D. Vande Ginste, "Rigorous full-wave resistance and inductance computation of 3-D interconnects," IEEE Microw. Wireless Compon. Lett., vol. 28, no. 6, pp. 455-457, Jun. 2018.

[5] M. Huynen, D. De Zutter, and D. Vande Ginste, "Broadband full-wave BIE impedance characterization of 3-D interconnects," in 2018 IEEE 27th Conference on Electrical Performance of Electronic Packaging and Systems (EPEPS), Oct. 2018, pp. 241-243.

[6] M. Huynen, M. Gossye, D. De Zutter, and D. Vande Ginste, "A 3-D differential surface admittance operator for lossy dipole antenna analysis," IEEE Antennas Wireless Propag. Lett., vol. 16, pp. 1052 $1055,2017$.

[7] J. Van Bladel, Electromagnetic Fields. John Wiley \& Sons, 2007.

[8] I. Gradshteyn and I. Ryzhik, Table of Integrals, Series, and Products. Academic Press, 2007.

[9] R. Collin, Field Theory of Guided Waves. IEEE Press, 1991.

[10] Y. Zhao, F. Ling, and J. Mao, "Novel Surface Impedance Modeling for Broadband Parameter Extraction of 3-D Interconnects," IEEE Microw. Wireless Compon. Lett., vol. 27, no. 1, pp. 7-9, Jan. 2017.

[11] Z.-G. Qian and W. C. Chew, "Fast full-wave surface integral equation solver for multiscale structure modeling," IEEE Trans. Antennas Propag., vol. 57, no. 11, pp. 3594-3601, Nov. 2009.

[12] A. C. Yucel, I. P. Georgakis, A. G. Polimeridis, H. Bağc1, and J. K. White, "VoxHenry: FFT-accelerated inductance extraction for voxelized geometries," IEEE Trans. Microw. Theory Techn., vol. 66, no. 4, pp. 1723-1735, Apr. 2018.

[13] Computer Simulation Technology. (2017) CST Microwave Studio. [Online]. Available: https://www.cst.com/

[14] Ansys Inc. (2015) ANSYS HFSS. [Online]. Available: https://www.ansys.com/ 